\title{
Modeling the Effect of Laser Heating on the Strength and Failure of 7075-T6 Aluminum
}

\author{
J.N. Florando*, J. D. Margraf, J. F. Reus, A.T. Anderson, R.C. McCallen, M.M. LeBlanc., J. R. Stanley, A.M. \\ Rubenchik, S. S. Wu, and W. H. Lowdermilk \\ Lawrence Livermore National Laboratory, Livermore, CA 94550 \\ *Corresponding author, email: florando1@IInl.gov
}

\begin{abstract}
The effect of rapid laser heating on the response of 7075-T6 aluminum has been characterized using 3-D digital image correlation and a series of thermocouples. The experimental results indicate that as the samples are held under a constant load, the heating from the laser profile causes non-uniform temperature and strain fields, and the strain-rate increases dramatically as the sample nears failure. Simulations have been conducted using the LLNL multi-physics code ALE3D, and compared to the experiments. The strength and failure of the material was modeled using the Johnson-Cook strength and damage models. In order to capture the response, a dual-condition criterion was utilized which calibrated one set of parameters to low temperature quasi-static strain rate data, while the other parameter set is calibrated to high temperature high strain rate data. The thermal effects were captured using temperature dependent thermal constants and invoking thermal transport with conduction, convection, and thermal radiation.
\end{abstract}

\section{Introduction}

With the increasing availability of commercial high power laser systems, there is growing interest in understanding the behavior of materials under laser irradiation. There has been considerable work, especially with regard to fire protection, on understanding how structural metals deform under transient tensile conditions[1-3], where the samples are held at a constant load and the strain is measured as the temperature is increased. In those experiments, however, the entire cross section is heated to a constant temperature, and the rate of heating is slow to allow for thermal equilibrium. There has also been work that investigated the effect of rapidly heating steel and Inconel in a constant load environment[4]. While this loading configuration is similar to that experienced during laser heating, the assumption is that the strain is uniform in the gage length. In contrast, with laser heating, the nonuniformity in the intensity profile of the laser causes temperature and strain variation within the gage section. In addition, the rapid temperature rise associated with laser heating can lead to significant changes in the local strain rate.

Due to the added complexities associated with laser heating experiments, additional diagnostic and computational tools are needed to help understand and capture the physics occurring in these experiments. In this study, 3-D digital image correlation, which provides the temporal and spatial resolution to measure the full-field strains, is coupled with a series of thermocouples to measure the 
temperature profile. On the computational side, a Lawrence Livermore National Laboratory (LLNL) multi-physics code, ALE3D, is used to couple the laser deposition, heat transport, material deformation, and material failure all in one code, with the goal of validating the code and models by comparison with the experimental observations.

While most of the work performed on transient behavior has been on steels, the focus of this work is on high strength aluminum alloys, specifically 7075-T6, which is an alloy commonly used in the aerospace industry. There have been a number of studies on the temperature and strain rate dependent properties of aluminum alloys under constant temperature conditions[5-8]. It was found that the temperature and strain rate dependency on the strength can be significant and non-linear, especially at the higher temperatures. Due to these dependencies, understanding how these alloys behave under high rate transient tensile conditions is still an open question.

\section{Experimental Procedure}

Tensile dog bone samples were fabricated from 7075-T6 clad aluminum sheet (Alclad) purchased from Castle Metals (Kent, WA). The gage section of the dog bone samples is $46 \mathrm{~mm}$ long by $10 \mathrm{~mm}$ wide by either $1 \mathrm{~mm}$ or $1.6 \mathrm{~mm}$ thick as shown in Figure 1 . Samples were tested at room temperature at a strain rate of $1 \times 10^{-3} \mathrm{~s}^{-1}$ to evaluate the room temperature isothermal properties. Based on these results, subsequent samples were first loaded to either $50 \%$ or $75 \%$ of the quasi-static yield stress, held at constant load, and then irradiated with the laser until the sample failed. The loading profile was chosen to examine the effect of loads on the observed behavior. A diode laser operating at $780 \mathrm{~nm}$ with a maximum power of $5 \mathrm{~kW}$ was used. For these experiments, a spot size of approximately $40 \mathrm{~mm}$ by 10 $\mathrm{mm}$ was irradiated at a power density of $50 \mathrm{~W} / \mathrm{cm}^{2}$. The laser intensity profile was measured by observing the relative intensity of the scattered light using a ccd camera by Point Grey. The absolute scale is determined by using a power meter to measure the total power. The intensity profile along the length of the sample is shown in Figure 2 . The profile across the width is determined to be constant. During the experiments, the temperature was measured using K-type 36 gage (0.005" diameter) thermocouples which were attached to the face of the sample opposite the side being irradiated by the laser. The thermocouples were attached at four locations, also shown in Figure $1 b$, along the length of the sample to acquire an estimate of the temperature distribution during the test. To examine the effect of thickness and initial loading, three different samples were laser irradiated as shown in Table 1.

The GOM Aramis 3-D digital image correlation (DIC) system was used to measure the full-field strains during the tests. The system uses two 5 megapixel cameras which were focused on the same side as the thermocouples. Rust-oleum ${ }^{\circledR}$ High Heat paint was used for the pattern, and was a white background painted on the surface followed by black dots to achieve a high contrast. Based on the pattern, the facet size was $25 \times 25$ pixels, and details regarding the measurement system can be found elsewhere [9].

\section{Simulation}

Continuum part-scale simulations were performed using LLNL's high performance production level computational tool called ALE3D (Arbitrary Lagrangian-Eulerian 3D and 2D) [10], which has unique 
physics and numerical models for application to laser damage modeling. The flexible and extensive code framework supports fully integrated hydrodynamics, heat transfer, solid and fluid dynamics, and chemistry models with the ability to capture the structural response for long (implicit) to short (explicit) time-scale applications.

The geometry in Figure 1 was preloaded in the simulations to the same load, as in the experiments, to simulate the condition before the laser heats the sample. The mesh size is approximately $\mathbf{3 0 0}$ micron, and for the pre loading conditions, a tensile stress boundary condition was applied to one end of the sample while the other end was fixed. The stress was scaled to produce a tensile stress equivalent to either $50 \%$ or $75 \%$ of the measured room temperature yield stress in the gage region of the sample, similar to the experimental conditions.

For the thermal part of the simulation, the heat deposition from the laser was simulated as a surface heat flux boundary condition, where the heat flux is proportional to the laser energy times the absorptivity. Since the absorptivity is dependent upon many factors including the surface roughness, as well as the temperature, the value of 0.3 for the absorptivity was used. This value is taken from a study on the same material with the same surface preparation, which also showed that the laser penetration is on the order of tens of nanometers thick, and can be treated as a surface flux [11]. The flux varies spatially and is based on the experimental profile shown in Figure 2. The flux is approximated using a cosine function and the match to the experiment is also shown in Figure 2. The laser intensity is varied in time to correspond to the laser being turned on after the sample has already reached a constant load. The thermal package allows for heat loss through conduction, convection and radiative thermal transport. Values for the temperature dependent heat capacity and thermal conductivity were taken from the military handbook[12], and the temperature dependent heat transfer convection coefficient was taken from the paper by Rubenchik et. al.[11], for convection on a horizontal plate.

Understanding that the mechanical response of aluminum alloys over a range of temperature and strain rates has many physical mechanisms occurring, for this study, the stress-strain behavior of the material is described using the empirical Johnson-Cook flow strength model, where the flow stress, $\sigma_{\text {flow }}$ can be described in the following manner[13]:

$\sigma_{\text {flow }}=\left(A+B \varepsilon^{n}\right)\left(1+C \ln \frac{\dot{\varepsilon}}{\dot{\varepsilon}_{o}}\right)\left(1-T^{* m}\right)+C_{p} p$

where

$T^{*}=\frac{T-T_{\text {room }}}{T_{\text {melt }}-T_{\text {room }}}$.

The parameter $\mathrm{A}$ is nominally the yield strength at the reference strain rate $\dot{\varepsilon}_{o}, \mathrm{~B}$ and $\mathrm{n}$ are the work hardening parameters, $C$ represents the strain rate dependence, $m$ is the temperature dependence, $C p$ is the pressure dependence, $T_{\text {melt }}$ is the melt temperature, and $T_{\text {room }}$ is the reference temperature, taken as the ambient room temperature. The main assumptions with the model are that the strain rate 
dependence will follow linearly with the log of the strain rate, and the temperature dependence will scale in a power law manner with $\mathrm{T}^{*}$. While other models, such as the Zerilli- Armstrong model[14], take into account the temperature dependence on the strain rate, the Johnson-Cook model was chosen due to its more extensive use in the community and the availability of parameters for common aluminum alloys.

A Johnson-Cook failure model[15] is used to describe the failure of the material, where the strain to failure can be described by:

$$
\varepsilon_{f}=\left(D_{0}+D_{D} \exp \left(D_{3} \sigma^{*}\right)\right)\left(1+D_{4} \ln \left(\frac{\dot{\varepsilon}}{\dot{\varepsilon}_{0}}\right)\right)\left(1+D_{5} T^{*}\right)
$$

where $\sigma^{*}$ is the stress triaxiality and is defined as

$$
\sigma^{*}=\frac{\sigma_{\text {mean }}}{\sigma_{\text {VonMises }}}
$$

The mean stress is the average of the three principal stresses

$$
\sigma_{\text {mean }}=\frac{\sigma_{1}+\sigma_{2}+\sigma_{3}}{3}
$$

and the Von Mises stress is

$$
\sigma_{\text {VonMises }}=\sqrt{\frac{\left(\sigma_{1}-\sigma_{2}\right)^{2}+\left(\sigma_{2}-\sigma_{3}\right)^{2}+\left(\sigma_{1}-\sigma_{3}\right)^{2}}{2}} .
$$

While empirical in nature, the physics behind the Johnson-Cook damage model is that the failure strain will depend on the stress triaxiality $\sigma^{*}$ in an exponential manner, modified by the constants $D_{D}$ and $D_{3}$. The term $\mathrm{D}_{0}$ represents the void nucleation term, and the model also has terms D4 and D5 that account for the strain rate and temperature dependence of the failure strain, respectively. The Johnson-Cook damage model is applicable to ductile materials where the failure mode is governed by the formation and coalescence of microvoids, which is strongly dependent on the stress state. In general, voids are more likely to nucleate and grow under conditions of high stress triaxiality leading to lower failure strains.

The simulation is run implicitly (implicit time integration) until the material localizes near failure. As the simulation approaches failure conditions, the time step drops precipitously and the code switches to the explicit time integration mode to capture failure. Damage in a material element is governed by the element damage parameter that follows the equation:

$$
D=\sum \frac{\Delta \varepsilon}{\varepsilon_{f}}
$$


where $\Delta \varepsilon$ is the incremental accumulation of strain in the element, and $\varepsilon_{f}$ is the failure strain. The material element is determined to have failed when the damage parameter in the element reaches unity. Once the failure criterion is reached, the code erodes (completely removes) the element, which simulates the generation and coalescences of voids.

\section{Results and Discussion}

An example of the DIC data for the thicker sample, Figure 3, shows the last frame before failure. The picture on the right shows a full-field pseudocolor map of the axial strains as well as the temperature at the four thermocouple locations attached to the sample. The strain map indicates that there is extensive strain localization, over $60 \%$ strain, in the center of the sample, and only a few percent strain near the $2^{\text {nd }}$ and $4^{\text {th }}$ thermocouples, even though the $2^{\text {nd }}$ and $4^{\text {th }}$ thermocouple locations are only 40 degrees colder. This behavior implies that there is an abrupt change in the temperature dependent strength of this alloy. The plot on the upper left of Figure 3 shows the stress as function of time, and the bottom left shows different strain contours at the different stages in time. The strain contours show the strain values in the sample along a user prescribed line, which appears in the pseudocolor plot on the right. The strain contour data shown is from the middle $14 \mathrm{~mm}$ of the sample. The strain data shows that during the early part of the experiment, the strain evolves slowly, and then increases rapidly near failure. The stages correspond to when a picture is taken, and the camera was set to take 2 frames/sec in the initial part of the experiment, and then increased to 15 frames/sec near failure.

The data outlined above was collected are for all of the test case samples. By examining the central portion of the dog bone geometry, where the highest strains occurred, temperature versus strain plots can be created for the three samples as shown in Figure 4. The samples held at $350 \mathrm{MPa}$ exhibit similar behaviors, and the sample held at $235 \mathrm{MPa}$ requires higher temperatures for a given strain, as expected. For all the samples very little strain occurs until the sample reaches a temperature where the strain levels begin to rapidly increase, which is around $210 \mathrm{C}$ for the $350 \mathrm{MPa}$ samples, and $260 \mathrm{C}$ for the 235 MPa sample. Since the behaviors are similar, the thicker $350 \mathrm{MPa}$ sample was chosen as an example to explore in greater detail.

The strain and strain-rate versus time plots for the $350 \mathrm{MPa}$ sample after the laser is turned on are shown in Figure 5. The strain rate is calculated as the instantaneous slope of the strain vs. time plot. The plots indicate that the strain rate is near quasi-static $\left(<10^{-1} / \mathrm{s}\right)$ in the beginning of the tests, but then increases dramatically after $60 \mathrm{sec}$, to a value near 500/s near failure.

In an effort to capture the strength and failure behavior in the simulations, the Johnson-Cook parameters, Table 2, were fit to the room temperature tensile stress-strain data, shown in Figure 5. To examine how these calibrated Johnson-Cook parameters compared to experimental data at different temperatures, the LLNL tool MIDAS[16] was used to plot the corresponding stress-strain relationship based on the calibrated model parameters, and compared to data from the aluminum handbook on 7075-T6 clad material. The comparison, shown in Figure 6, indicates that while the calibrated model matches the room temperature and $260 \mathrm{C}$ data, it underpredicts the strength at modest temperatures above room temperature, and over predicts the strength at higher temperatures. The reasoning is that 
initially the strengthening due to the $\mathrm{Zn}-\mathrm{Mg}$-Cu precipitates in 7075 -T6 are not sensitive to modest temperature increases, until a temperature where they begin to lose coherency, similar to over-aging[7, 17] . At that point, the strength can decrease rapidly. Since these types of mechanisms are not represented in the Johnson-Cook model it is not surprising that there are significant discrepancies as a function of temperature. In addition, due to the large strain rate changes near the end of the experiment, as shown in figure 4 , an understanding of the temperature dependency at high strain rates is also needed.

Lee et al. measured the high strain rate behavior of 7076-T6 from $25 \mathrm{C}-300 \mathrm{C}$ [8]. Their study showed that the strain rate sensitivity increases with increasing temperature, which is in agreement with our observations and has been seen by other studies on aluminum and aluminum alloys [18]. The higher rate sensitivity at higher temperatures is usually attributed to viscous drag mechanisms that occur at the higher strain rates, which implies there are several deformation mechanisms that are operating and dominant under different regimes of strain rate and temperature.

Since the Johnson-Cook model does not have the necessary parameters to describe this complex behavior, a dual-condition model implementation was used to account for the behavior. The implementation allows for a different set of Johnson-Cook parameters to describe the behavior in the two distinct regimes, low temperature quasi-static, and high temperature high strain rate. The start temperature, $T_{s}$, and the temperature range of the transition, $T_{\text {range, }}$ specifies the transition between the two model parameter sets. For this material, the $T_{s}$ is chosen to be $237 \mathrm{C}$, and $T_{\text {range }}$ is $10 \mathrm{~K}$. In the transition regime the strength is governed by a changing linear rule of mixtures of the two models. Table 2 lists the Johnson-Cook parameters used for the two different regimes. Figure 7 shows a comparison between the model parameters and the experimental data in their respective regimes. To model the damage in the material, Table 3 shows the implemented Johnson-Cook damage parameters that are from the literature[19]. One exception is the temperature dependence term, D5, was determined from an analysis of the experiment.

A comparison between the strain field from the simulation and the experiment is shown in Figure 8a. The overall size and shape match well, indicating that the models are able to capture the overall behavior. A more detailed comparison between the experimental strain contour at the last frame before failure and the corresponding strain contour from the simulations are shown in Figure $8 \mathrm{~b}$. The simulation appears to be over predicting the amount of strain, however, the peak strain and overall shape is similar. The higher strain values imply that the simulations are under predicting the strength, which can be expected since the simulations are calibrated to constant temperature tests, where the temperature in the sample is equilibrated before the tests are performed. During this stabilization, the sample has sufficient time to soften due to mechanisms such as grain growth, and coarsening of the precipitate. During the laser test, however, the temperature transients are much faster, which does not allow time for these additional softening mechanisms to occur. A comparison of the micrographs of the grip area, which experienced no heating, to the laser heated area in the middle of the sample, Figure 9 , shows no discernible difference in the grain size or precipitate size after the laser experiment, even though the middle of the sample reached temperatures over $250 \mathrm{C}$. In addition, nanoindentation hardness measurements show similar hardness in the two regions with an average hardness of $2.4 \mathrm{GPa}$ 
$\pm 0.1 \mathrm{GPa}$ in middle region, and $2.5 \mathrm{GPa} \pm 0.1 \mathrm{GPa}$ in the grip region. The lack of significant differences between the two regions indicates that the time span is sufficiently small to prevent significant microstructural changes from occurring.

A comparison of the temperature versus time plots between the simulations and experiments are shown in Figure 10. There are two distinct zones in the temperature rise. At early times, the temperature is mostly dependent on the heat capacity of the material and the initial heat flux from the laser. At longer times, the thermal loss components, including conduction, convection, and radiative cooling effect the shape of the temperature time profile. The simulation results are very close to the experimental thermocouple data, signifying that thermal constants are adequate in capturing the thermal history during the laser experiments.

\section{Conclusions}

Samples of 7075-T6 clad have been tested under rapid transient tensile experiment conditions via laser heating. Utilizing 3-D image correlation and a series of thermocouples to measure the full-field strains and temperatures respectively, the experimental results show that the strain localizes in the center region, even though the temperature profile is more diffuse. In addition, the experimental results show that the strain rate is rapidly changing leading to a local strain rate of $\sim 500 / \mathrm{s}$ near the point of failure. These results can be captured in the simulations by implementing a model that allows for two sets of strength parameters: one that describes the low temperature quasi-static regime, and one that captures the high temperature high strain rate behavior. The temperature profile can also be matched by using temperature dependent material properties, as well as appropriate heat boundary conditions.

\section{Acknowledgement}

The authors would like to thank Nathan Barton for implementing the dual condition model in ALE3D. Funding for this work is through the LLNL Laboratory Directed Research and Development (LDRD) Program. This work was performed under the auspices of the U.S. Department of Energy by Lawrence Livermore National Laboratory under Contract DE-AC52-07NA27344. 


\section{References}

1. J. Chen and B. Young, Stress-strain curves for stainless steel at elevated temperatures, Eng. Struct. 28, 229-239 (2006).

2. J. Outinen, et al., Fire design model for structural steel $\mathrm{S} 355$ based upon transient state tensile test results, J. of Constr. Steel Res. 42, 161-169 (1997).

3. J. Maljaars, et al., Constitutive model for aluminum alloys exposed to fire conditions, Metall. and Mater. Trans. A 39, 778-789 (2008).

4. $\mathrm{H}$. Jones and $\mathrm{K}$. $\mathrm{Wu}$, Tensile instability and deformation behavior of rapidly heated metals in a constant-load environment, Intern. J. of Thermophys. 11, 675-691 (1990).

5. C. Moosbrugger, Atlas of stress-strain curves, ASM International, Materials Park (2002).

6. Y. Lin, et al., Hot compressive deformation behavior of $7075 \mathrm{Al}$ alloy under elevated temperature, J. of Mater. Sci. 47, 1306-1318 (2012).

7. J.-F. Li, et al., Mechanical properties, corrosion behaviors and microstructures of 7075 aluminium alloy with various aging treatments, Trans. of Nonferr. Metal. Soc. of China 18, 755-762 (2008).

8. W.-S. Lee, et al., The strain rate and temperature dependence of the dynamic impact properties of 7075 aluminum alloy, J. of Mater. Process. Technol. 100, 116-122 (2000).

9. M. LeBlanc, et al., Image correlation applied to single crystal plasticity experiments and comparison to strain gage data, Exp. Tech. 30, 33-37 (2006).

10. A. Nichols, editor, ALE3D An Arbitrary Lagrange/Eulerian 2D and 3D Code System, LLNLSM-642812. (2013).

11. A. M. Rubenchik, et al., Temperature-dependent 780-nm laser absorption by engineering grade aluminum, titanium, and steel alloy surfaces, Opt. Eng. 53, 122506-122506 (2014).

12. M. Handbook, MIL-HDBK-5H: Metallic Materials and Elements for Aerospace Vehicle Structures, US Department of Defense 3-244 (1998).

13. G. R. Johnson and W. H. Cook, Proceedings of the 7th International Symposium on Ballistics,21, 541-547 (1983).

14. F. J. Zerilli and R. W. Armstrong, Dislocation mechanics based constitutive relations for material dynamics calculations, J. of Appl. Phys. 61, 1816-1825 (1987).

15. G. R. Johnson and W. H. Cook, Fracture characteristics of three metals subjected to various strains, strain rates, temperatures and pressures, Eng. Fract. Mech. 21, 31-48 (1985).

16. M. Tang, et al., MIDAS (Material Implementation, Database, and Analysis Source): A comprehensive resource of material properties, LLNL-PROC-464065 (2010).

17. J. Park and A. Ardell, Microstructures of the commercial $7075 \mathrm{Al}$ alloy in the T651 and T7 tempers, Metall. Trans. A 14, 1957-1965 (1983).

18. J. Seidt and A. Gilat, Plastic deformation of 2024-T351 aluminum plate over a wide range of loading conditions, Int. J. of Solids and Struct. 50, 1781-1790 (2013).

19. N. Brar, et al., Constitutive model constants for Al7075-T651 and Al7075-T6, AIP Conference Proceedings 1195, 945 (2009). 
Figures and Tables

Table 1.Summary of experimental conditions

\begin{tabular}{|c|c|c|}
\hline Sample & $\begin{array}{c}\text { Thickness } \\
(\mathrm{mm})\end{array}$ & $\begin{array}{c}\text { Constant } \\
\text { Stress (MPa) }\end{array}$ \\
\hline 1 & 1.6 & 350 \\
\hline 2 & 1 & 350 \\
\hline 3 & 1 & 235 \\
\hline
\end{tabular}

Table 2. Johnson-Cook Strength Parameters

\begin{tabular}{|c|c|c|c|c|c|c|c|}
\hline & $\mathrm{A}(\mathrm{MPa})$ & $\mathrm{B}(\mathrm{MPa})$ & $\mathrm{n}$ & $\mathrm{C}$ & $\mathrm{m}$ & $\mathrm{T}_{\text {melt }}(\mathrm{K})$ & $\mathrm{C}_{\mathrm{p}}$ \\
\hline $\begin{array}{c}\text { Single } \\
\text { condition }\end{array}$ & 480 & 370 & 0.43 & 0.0072 & .74 & 750 & 0 \\
\hline $\begin{array}{c}\text { "Multi- } \\
\text { condition" } \\
\text { Low Temp }\end{array}$ & 520 & 400 & 0.43 & 0.015 & 1.5 & 750 & 0 \\
\hline $\begin{array}{c}\text { "Multi- } \\
\text { Condition" } \\
\text { High Temp }\end{array}$ & 430 & 370 & 0.43 & 0.035 & 1.2 & 750 & 0 \\
\hline
\end{tabular}

Table 3. Johnson-Cook Damage Parameters

\begin{tabular}{|c|c|c|c|c|c|}
\hline $\mathrm{D}_{\mathrm{o}}$ & $\mathrm{D}_{\mathrm{D}}$ & $\mathrm{D}_{3}$ & $\mathrm{D}_{4}$ & $\mathrm{D}_{5}$ & $\dot{\varepsilon}_{\mathrm{o}}$ \\
\hline-0.068 & 0.45 & -0.95 & 0.036 & 6 & $1 / \mathrm{s}$ \\
\hline
\end{tabular}

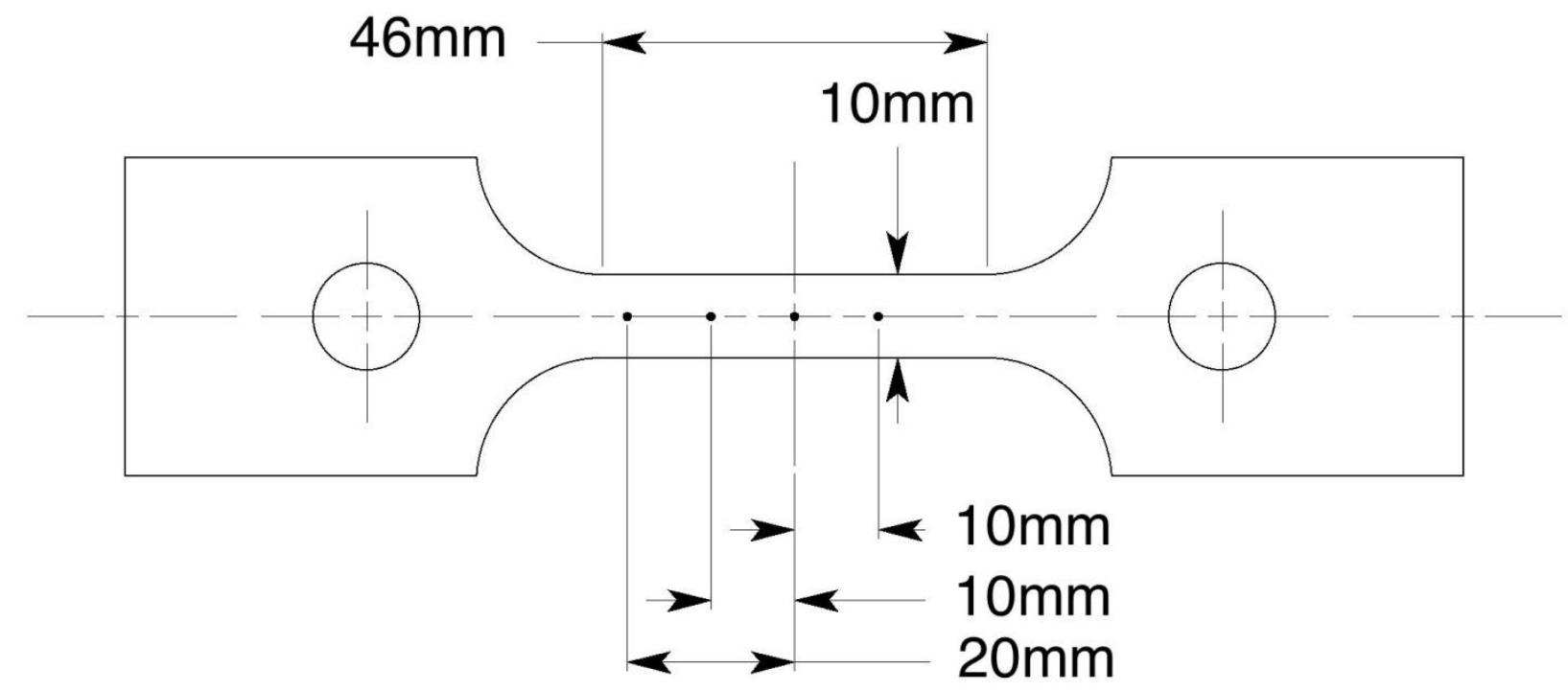

Figure 1- Tensile sample dimensions and location of thermal couples (all dimensions in $\mathrm{mm}$ ) 


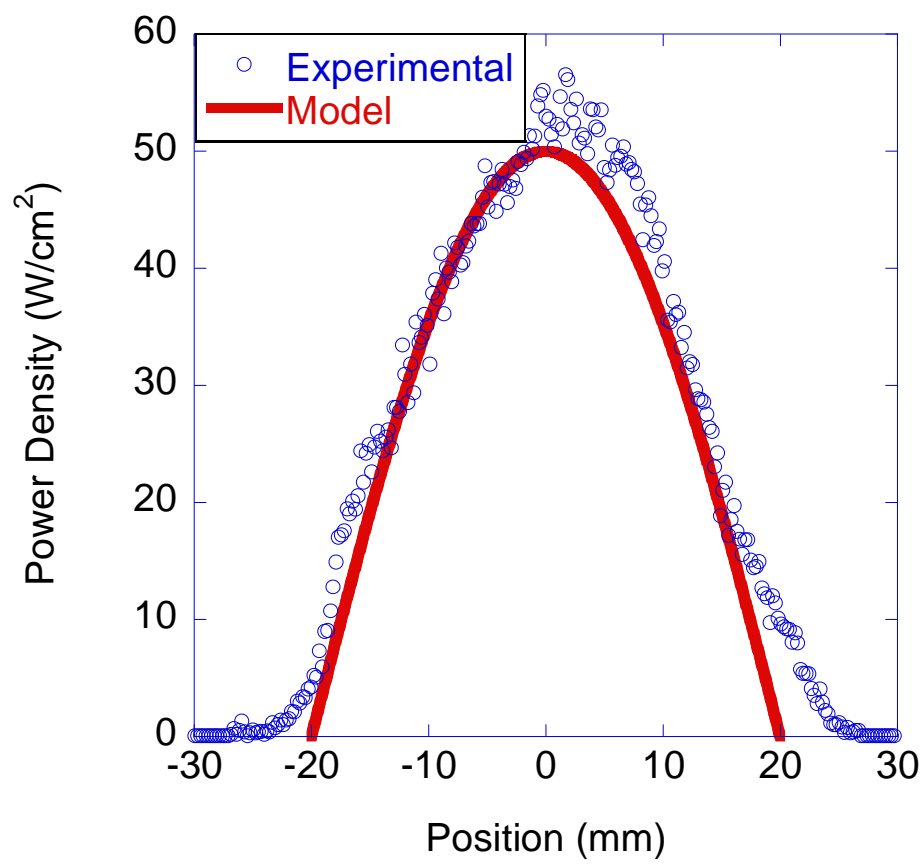

Figure 2-Comparison of the measured laser beam intensity profile along of the length of the sample to the cosine distribution used in the simulations.
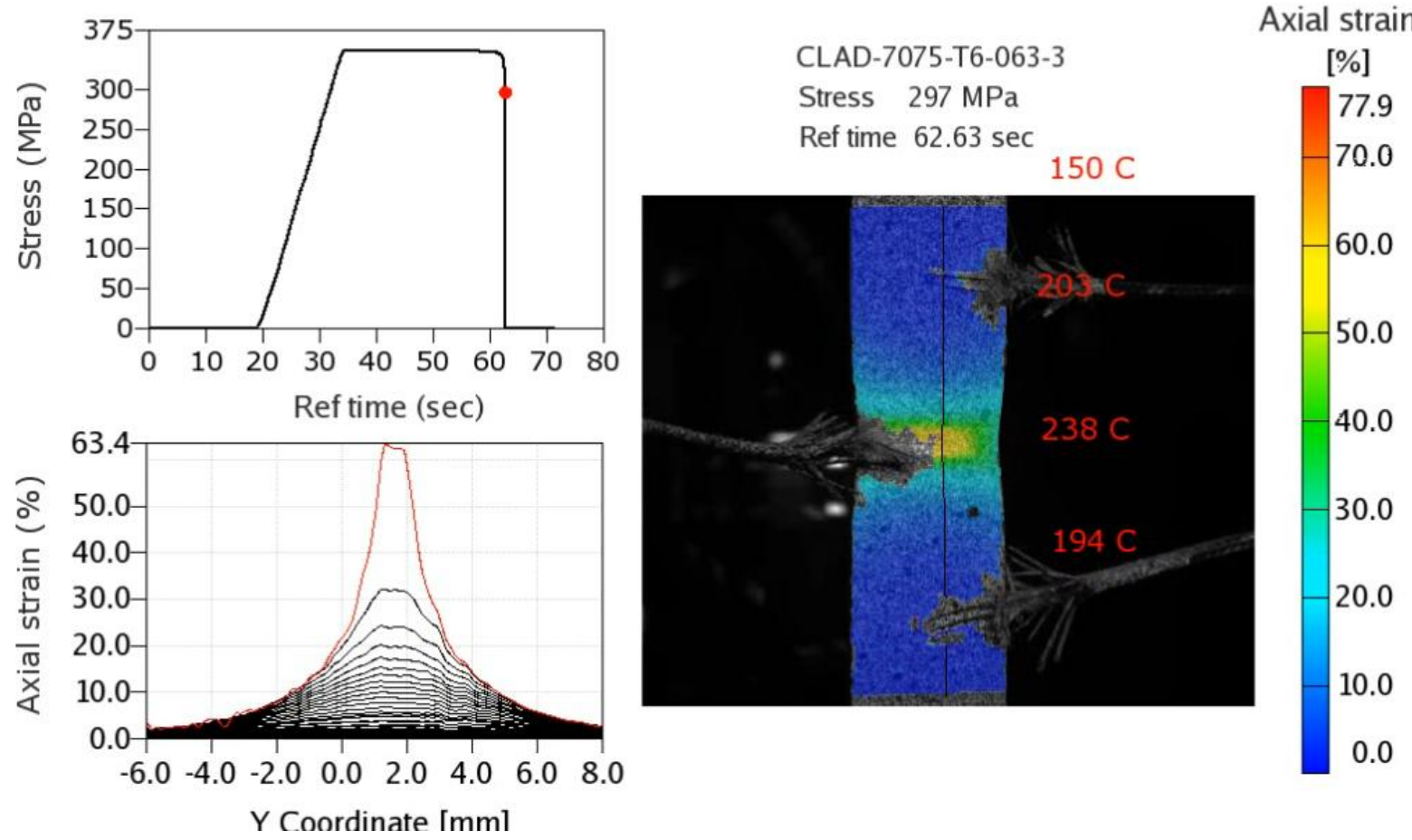

Figure 3- Experimental stress, temperature, and full-field image correlation data. The strain contours shown are reported at a rate of $2 /$ second until a peak strain of $2 \%$, at which the frequency is increased to 14 /second at failure. 


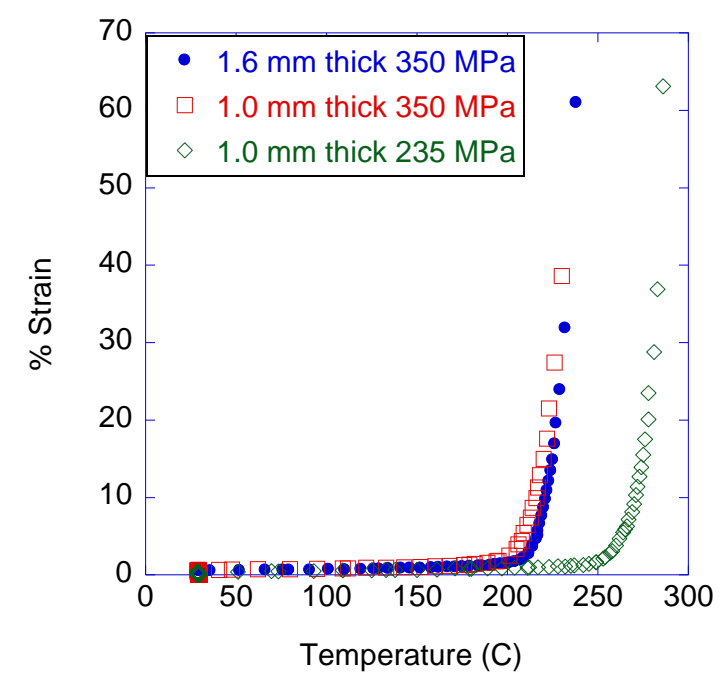

Figure 4- Experimental temperature versus strain plots for the three samples, where the strain is taken from the DIC data at the middle of the sample.

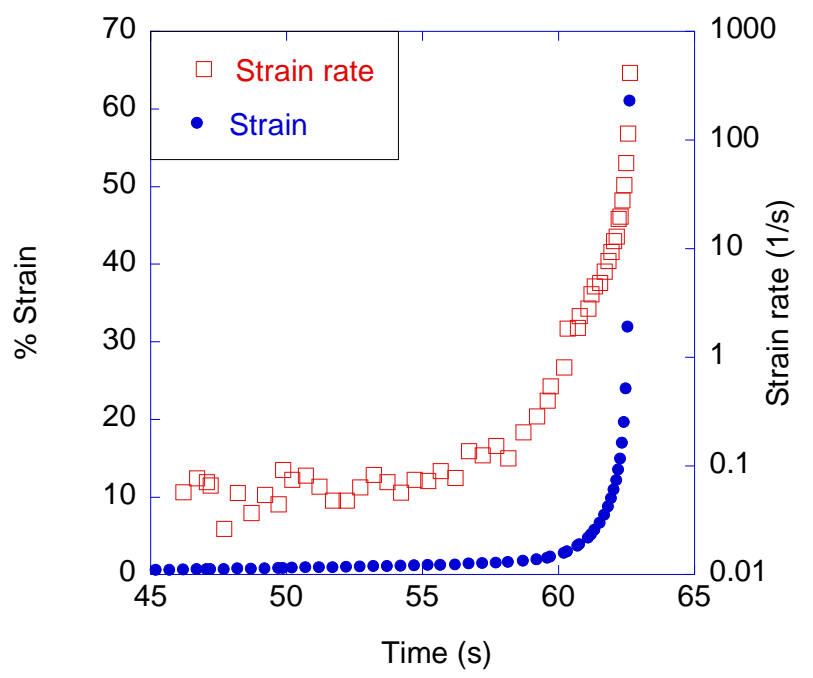

Figure 5 - Strain and strain rate versus time where the strain is taken from the DIC data at the middle of the sample. 


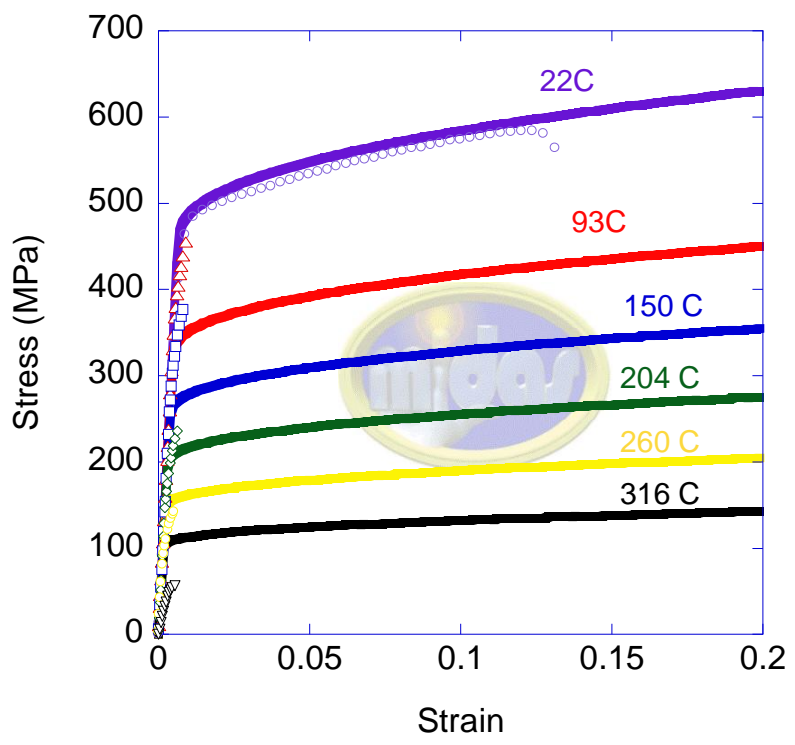

Figure 6- Comparison of experimental data with model fit for "single" condition model. The data is represented as markers, and the lines are the simulations results. The model under predicts the strength just above room temperature, and under predicts the strength at higher temperatures.
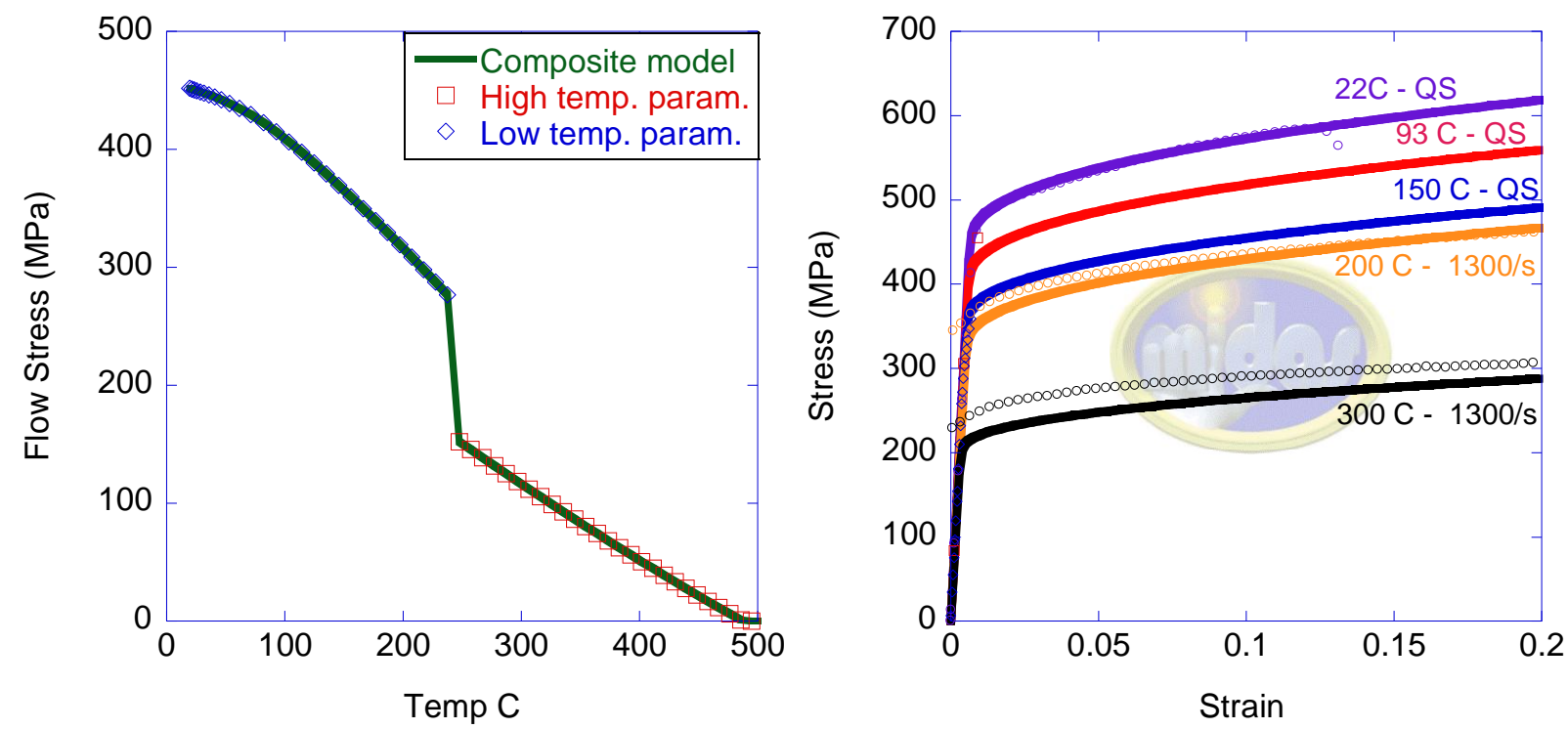

Figure 7- Comparison of the experimental data with the "dual-condition" model fits in the two regimes. The data is represented as markers, and the lines are the simulations results. The low temperature parameters are calibrated to the lower temperature quasi-static data, and the high temperature parameters are calibrated to the higher temperature high strain rate data. The transition begins around $237 \mathrm{C}$ and completes by $247 \mathrm{C}$. 


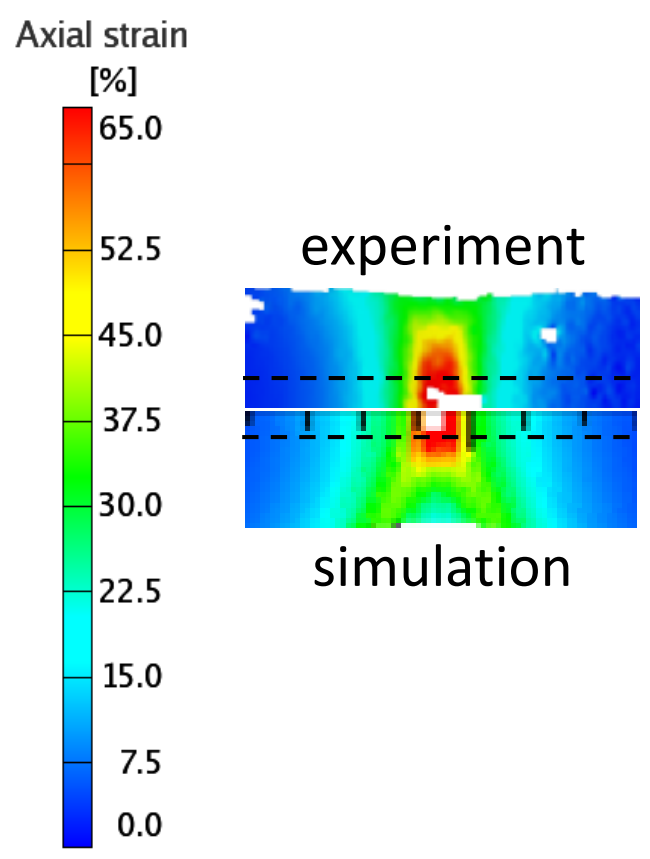

a)

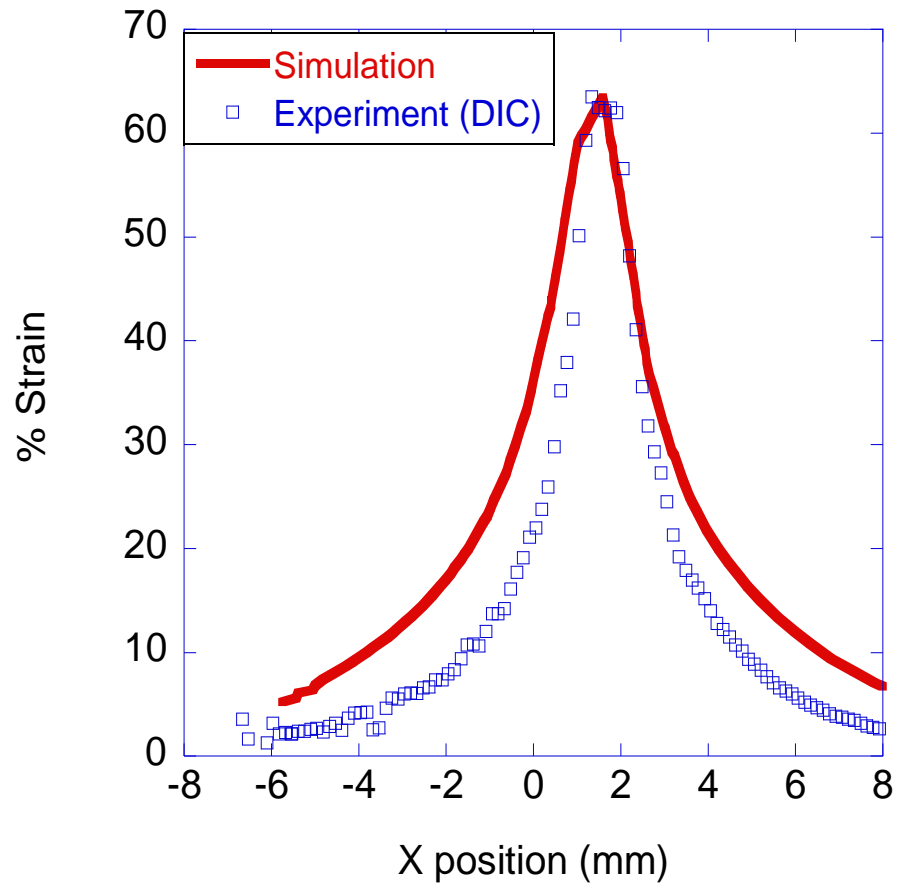

b)

Figure 8-Comparison of the a) full-field strains and b) the strains along the dash line, of the DIC data and the simulation results right before failure.

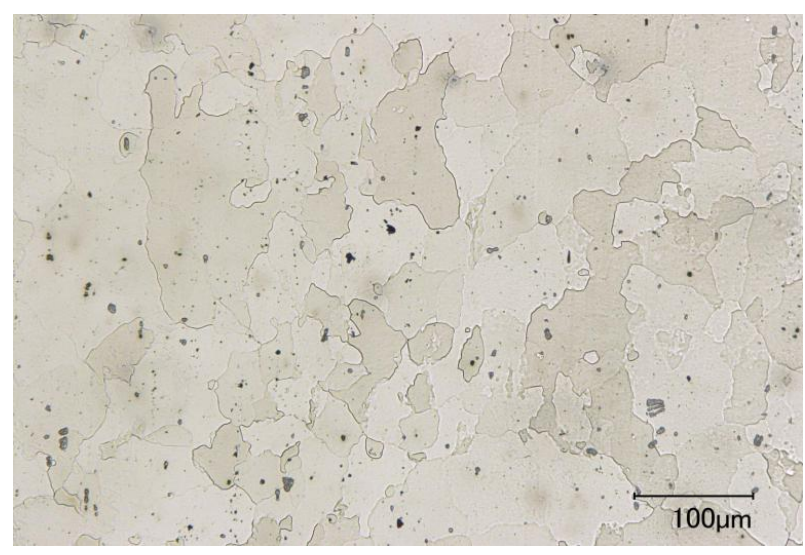

a)

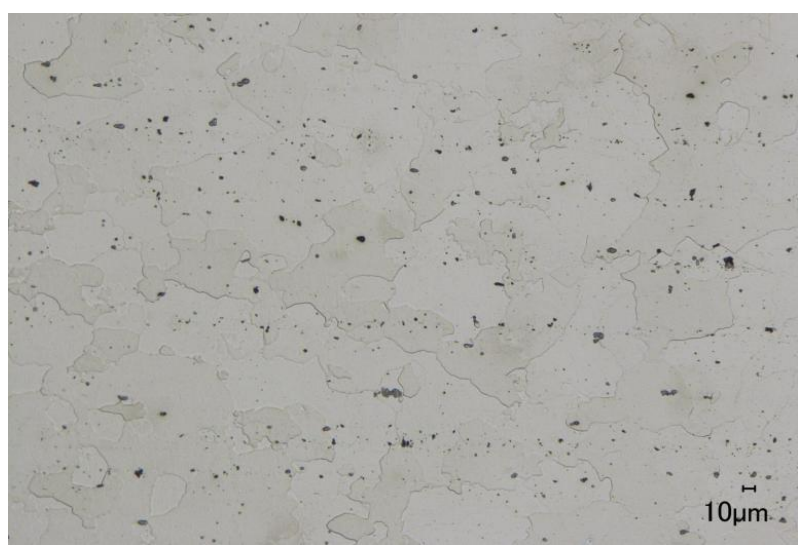

b)

Figure 9- Micrograph of the material from a) grip area far away from the laser irradiation region, and b) near middle of the sample where the laser irradiation is the highest. There are no discernible differences in the microstructure between the two regions despite the large temperature changes experience irradiated region. 


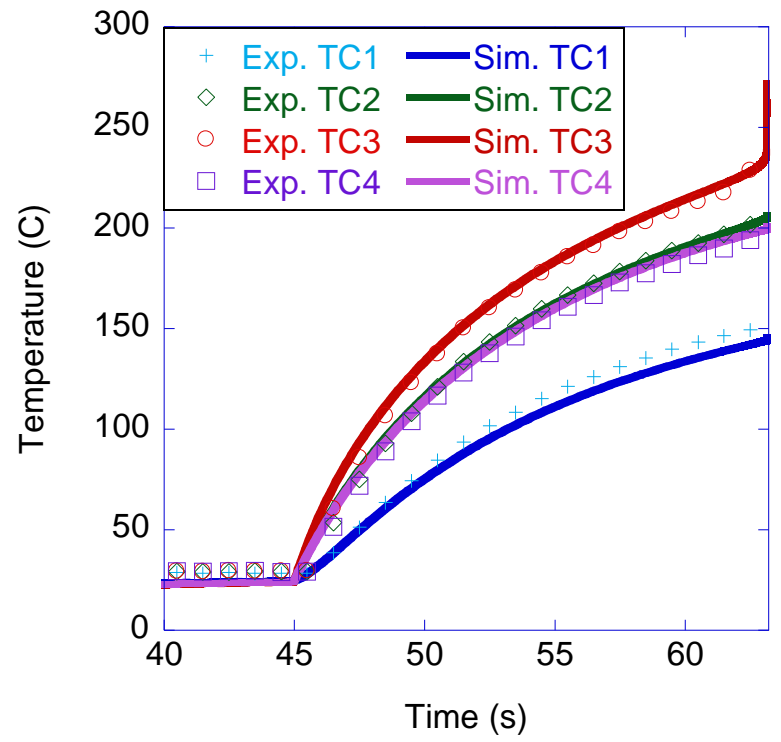

Figure 10-Comparison of the experimental and simulation temperature versus time profiles. 